\title{
Le retour d'expérience des incidents et son exploitation
}

\author{
C. BATAILLE ${ }^{1}$, C. LEFAURE ${ }^{1, a}$, G. ABÉLA ${ }^{2}$, P. BONAVENTURE ${ }^{3}$, \\ P. BRUNET ${ }^{4}$, F. BOSLE ${ }^{5}$, S. ISRAËL $^{6}$, L. HIDALGO $^{7}$, \\ J.-Y. MARIE ${ }^{5}$, J.-P. PATOUX ${ }^{8}$, J.-C. POULAIN ${ }^{9}$, P. RUEL ${ }^{10}$, \\ J.-P. SERVENT ${ }^{11}$, V. SOARES ${ }^{12}$
}

RÉSUMÉ Établi dans le cadre du groupe de travail COFREND-SFRP, cet atelier avait pour principal objectif de recenser le retour d'expérience des incidents existant dans le domaine de la gammagraphie industrielle et, si possible, de l'améliorer. Les produits issus de l'atelier sont principalement destinés à enrichir le contenu des formations des opérateurs et des personnes compétentes en radioprotection des entreprises de gammagraphie industrielle. Différentes sources bibliographiques dans lesquelles des incidents/accidents sont recensés ont été analysées : les bases de données IRID et RELIR ont été particulièrement étudiées. Il a alors été identifié que le retour d'expérience existant n'était pas totalement représentatif des événements rencontrés en France aujourd'hui. De nouveaux types d'incidents (non maîtrise de la zone balisée, blocage du porte-source dans la gaine dû à la présence de corps étranger...) ont été identifiés à partir desquels de nouvelles fiches RELIR ont été établies. En parallèle, une synthèse des leçons issues des incidents recensés au cours de l'atelier a été élaborée.

ABSTRACT Feedback experience from incidents and its use.

This working group aimed at listing and, if possible, improving feedback experience from incidents in the field of gamma radiography. The documents produced by the group may be used to enrich the content of training courses addressed to operators or health physicists from gamma radiography firms. The participants analysed different bibliographical sources where incidents/accidents were presented: the incident databases named IRID and RELIR were particularly studied. It was then identified that the existing feedback experience was not representative from the events occurring today in France. New types of incidents (no mastery of the demarked area, source stuck due to foreign bodies in the guide tube) were thus identified from which new RELIR publications were established. In parallel,

\footnotetext{
Animateur

Centre d'Étude sur l'Évaluation de la Protection dans le domaine Nucléaire (CEPN), 28 rue de la Redoute, 92260 Fontenayaux-Roses, France.

EDF, Division Ingénierie Nucléaire, 1 place Pleyel, 93282 Saint-Denis Cedex, France.

EDF, CEIDRE, 1 place Pleyel, 93282 Saint-Denis Cedex, France.

SGS Qualitest Industrie, 191 avenue Aristide Briand, 94237 Cachan Cedex, France.

Norisko Équipements, 37 rue des frères Lumières, 69680 Chassieu, France.

CEGELEC NDT, Z.I. du Bois des Bordes, Le Plessis Pâté, 91229 Brétigny-sur-Orge Cedex, France.

APAVE, 97 bd Victor Hugo, 93400 Saint-Ouen, France.

Gaz De France, DGI - Division SIQE, 5 rue F. de Lesseps, BP 50559, 60205 Compiègne Cedex, France.

CRAM Normandie, 3 place des Faïenciers, Saint Sever, 76100 Rouen, France.

10 Institut de Soudure Industrie, Z.I. Paris Nord 2, 90, rue des Vanesses, 93420 Villepinte, France.

11 INRS/ECT, 30 rue Olivier Noyer, 75680 Paris Cedex 14, France.

12 SIRAC Centre Technique, 25 Rue Claude Bernard, 78310 Maurepas, France.
} 
a synthesis of the lessons learned from the incidents listed during the work was elaborated.

Keywords: incident analysis / radiography

\section{Introduction}

L'un des moyens les plus efficaces pour réduire le nombre et la gravité des incidents radiologiques est d'utiliser à des fins pédagogiques et de sensibilisation le retour d'expérience des incidents passés concernant des travailleurs. Ainsi, cet atelier avait pour principal objectif de recenser le retour d'expérience des incidents existant dans le domaine de la gammagraphie industrielle et, si possible, de l'améliorer. Les produits issus de l'atelier sont principalement destinés à enrichir le contenu des formations des opérateurs et des personnes compétentes en radioprotection des entreprises de gammagraphie industrielle.

L'atelier, animé par le CEPN, s'est réuni à deux reprises pendant la durée du groupe de travail. Il était composé de représentants de différents organismes : opérateurs de gammagraphie (Norisko, Institut de Soudure, SGS Qualitest Industrie), constructeurs d'appareils de gammagraphie (Cegelec), donneurs d'ordre (EDF CEIDRE, GDF), organismes de contrôle et de recherche (APAVE, CRAMNormandie, INRS).

Cet article présente le déroulement de cet atelier ainsi que ses résultats.

\section{1. Étude de sources bibliographiques de retour d'expérience des incidents dans le domaine des tirs gammagraphiques}

La première étape a consisté en une présentation de bases de données connues relatant des incidents dans le domaine de la gammagraphie et de différentes sources bibliographiques. Les bases de données IRID (Ionising Radiation Incident Database - Royaume-Uni) et RELIR (Retour d'Expérience sur Les Incidents Radiologiques - France) ont notamment été analysées.

\subsection{La base de données IRID}

IRID a été développée en Grande-Bretagne à partir de 1996 par le National Radiation Protection Board (NRPB) ${ }^{13}$, le Health and Safety Executive (HSE) et

13 Intégré dorénavant dans l'Agence de Protection Sanitaire (Health Protection Agency). 
l'Agence de l'environnement britannique (Croft et al., 1999). Ses objectifs sont les suivants :

- Étudier les accidents radiologiques particulièrement dans le domaine de l'industrie non nucléaire, la médecine, la recherche et l'enseignement.

- Fournir, à travers des publications appropriées, un retour d'expérience et des conseils aux acteurs de la prévention, et limiter les conséquences des éventuels nouveaux accidents.

- Établir « un patrimoine de sécurité radiologique » dans des secteurs d'activités où la radioprotection n'est pas la priorité.

Selon IRID, « un incident radiologique est un événement non prévisible pouvant résulter d'une erreur humaine, d'une déficience de l'équipement ou d'une défaillance du système de management ».

Dans la base de données, un tiers des incidents concerne la gammagraphie industrielle (26 cas sont recensés). Ils sont relatifs à des pertes de sources, des sources endommagées (corrosion des conteneurs et fuites), des défauts de maintenance, des équipements défectueux ou à l'absence de port de dosimètres.

Une analyse de la base de données IRID semble montrer que des thèmes communs sont à retenir pour les incidents :

- Défaut du système de management : de mauvaises règles internes, un mauvais contrôle du personnel, des défaillances dans l'utilisation des instruments de mesures résultent d'un manque d'efficacité du système managérial.

- Absence ou mauvaise utilisation des appareils de mesure : un des facteurs contribuant à la majeure partie des incidents est l'absence d'utilisation ou la mauvaise utilisation des appareils de mesure. Les procédures doivent contenir suffisamment d'informations sur les contrôles radiologiques à effectuer. Elles doivent aussi définir les instruments de contrôle à utiliser, le niveau de formation des utilisateurs et les techniques de maintenance de ces appareils.

- Défaut de formation des travailleurs utilisant des sources : de nombreux incidents surviennent par manque de connaissances ou par une mauvaise formation / information du personnel.

\subsection{Le réseau RELIR}

RELIR est un système français de retour d'expérience sur les incidents radiologiques, dont l'objectif est de recueillir des incidents « exemplaires » dans différents secteurs d'activités (industriel, médical et vétérinaire, recherche et enseignement et transports), afin qu'ils soient présentés, sous forme de fiches pédagogiques, dans des sessions de formations professionnelles à la radioprotection (Vaillant et al., 2006). Il a été créé, en 2001, par la Section des personnes compétentes de la SFRP, avec la collaboration de l'IRSN, l'INRS, l'INSTN, le CEPN et de l'Institut Curie. 
Dans RELIR, est considéré comme incident «toute situation, événement, suite d'événements, comportement, anomalie... susceptible de générer (ou ayant effectivement généré) une exposition professionnelle non maîtrisée ou une contamination ».

Le système RELIR s'appuie sur un réseau d'acteurs de la radioprotection : les « modérateurs ». Lorsqu'une personne est témoin d'un incident ou concernée par cet incident (personne compétente, salarié, médecin du travail...), elle a la possibilité de joindre le modérateur du domaine d'activité professionnelle correspondant. Le modérateur remplit alors un questionnaire-type qui permet de décrire l'incident, et d'en identifier et analyser les causes et les conséquences. À partir des différents incidents recensés, les modérateurs sélectionnent de façon collégiale les cas les plus intéressants en vertu de leur intérêt pédagogique et établissent des fiches anonymisées présentant chaque incident retenu et les leçons qui peuvent en être tirées. Les fiches sont alors mises en ligne sur le site internet RELIR (www. relir.cepn.asso.fr). À l'heure actuelle, environ 50 fiches sont publiées.

Avant l'atelier, quatre fiches se rapportaient à des incidents lors de tirs gammagraphiques (blocage de la source dans la gaine d'éjection, incident lors du changement de source, sortie de source non maîtrisée...).

\subsection{Les fiches publiées dans la lettre d'information du réseau ALARA Européen (EAN)}

Chaque semestre, le réseau ALARA Européen (EAN) publie une lettre d'information dans laquelle est présenté un incident/accident récent. Plusieurs incidents de gammagraphie industrielle ayant eu lieu dans des pays européens ont ainsi été décrits et sont disponibles sur le site web du réseau EAN : www.eu-alara.net. Ils illustrent principalement le besoin de maintenance des gammagraphes, de formation des personnels et de prise en compte des activités connexes lors d'un contrôle.

Il est à noter que le réseau EAN a organisé, en 2001, un séminaire intitulé « Radiographie industrielle - Améliorations dans le domaine de la radioprotection ». À cette occasion, il a été recommandé que les formations incluent des exercices pratiques ainsi que l'étude de retour d'expérience d'incidents/accidents (EAN, 2001). Dans cette optique, la remontée des incidents au travers de mécanismes de reporting efficaces et anonymes, aux niveaux national (tels que IRID ou RELIR), européen et international a été encouragée.

\subsection{Les documents de l'Agence internationale pour l'énergie atomique}

L'Agence internationale pour l'énergie atomique a publié différents documents relatifs à la radioprotection dans le domaine de la gammagraphie (AIEA, 1998, 
1999). Il est reporté que les principales causes des incidents/accidents en gammagraphie industrielle sont les suivantes :

- Inexistence ou déficit de contrôle réglementaire (pas de vérification de l'état des appareils, des procédures de travail, de la qualification du personnel...).

- Non respect des procédures de travail.

- Manque de formation des opérateurs.

- Défaut de maintenance.

- Erreur humaine.

- Défaillance technique.

Les principales leçons concernent :

- La nécessité de disposer et de respecter les procédures de sûreté lors des contrôles.

- L'intérêt de bénéficier d'inspections réglementaires (équipements, procédures, formation et information du personnel).

- L'importance de former et d'informer le personnel périodiquement (et de contrôler les connaissances acquises).

\section{Analyse du retour d'expérience disponible}

L'analyse critique des sources de retour d'expérience (REX) existantes a montré que le REX international (par exemple, issu de IRID) peut ne pas être adapté au cas français : chaque pays utilise en effet des gammagraphes différents, répondant à des réglementations plus ou moins sévères.

Compte tenu de ce constat, les réflexions du groupe se sont focalisées sur le matériel disponible dans RELIR. Il a alors été identifié que le REX existant (et les fiches RELIR) n'était pas totalement représentatif des événements rencontrés en France aujourd'hui et que toutes les causes d'incidents n'étaient pas répertoriées. Par exemple, il a été mis en avant que la base RELIR ne couvrait que les secteurs non nucléaires et que la typologie des incidents rencontrés lors de contrôles dans les centrales nucléaires pouvait être différente.

Le REX existant a donc été enrichi grâce aux différentes expériences des participants de l'atelier.

\section{Enrichissement du retour d'expérience}

Au travers d'échanges et de différents exposés, plusieurs types d'incidents susceptibles de se produire aujourd'hui en France ont été identifiés. Les thèmes suivants ont été retenus. 


\subsection{Non maîtrise du balisage ou de la zone balisée lors d'un contrôle}

Ce thème regroupe plusieurs types d'incidents :

- «Incidents consécutifs à un balisage défaillant» : le balisage est défaillant, un accès n'est pas balisé et permet de pénétrer à l'intérieur de la zone d'exclusion. Le, ou les, individus peuvent alors se rapprocher de la source pendant le contrôle radiographique et ainsi être exposés au rayonnement.

- «Incidents consécutifs à une évacuation incomplète de la zone d'exclusion » : des intervenants se retrouvent «prisonniers » de la zone d'exclusion. Ceci est souvent le cas lorsque les contrôles/tirs se réalisent dans des bâtiments étendus où le personnel est nombreux et réalise des activités connexes.

- «Franchissement volontaire de balisage »: dans différentes circonstances, pour éviter de contourner la zone d'exclusion, pour faire une manœuvre, ou pour toute autre raison, des balisages de tirs radiographiques peuvent être franchis volontairement.

\subsection{Blocage du porte-source dû à la présence de corps étranger dans la gaine (sable, rouille...)}

Une anomalie fréquemment rencontrée est l'impossibilité de rentrer le portesource dans le gammagraphe. La présence d'un corps étranger (sable, rouille...) dans la gaine d'éjection est souvent à la source du blocage. Il a été souligné que les conditions de travail en gammagraphie industrielle sont souvent difficiles, voire hostiles et que malgré la robustesse des appareils, ceux-ci doivent être vérifiés régulièrement par les opérateurs au début des opérations (propreté et intégrité des branchements).

\subsection{Manque de formation des jeunes opérateurs}

Les participants ont souligné la nécessité de renforcer la formation des stagiaires et d'inclure dans les formations, des séances « comportement à adopter » face à l'incident.

\subsection{Prise en compte du facteur humain}

De nombreux incidents ont lieu de nuit ou dans des conditions de travail difficiles. Les participants ont insisté sur l'importance du facteur humain lors des contrôles. Il peut être nécessaire de limiter la charge de travail pour la rendre compatible avec l'attention nécessaire. L'utilisation d'outillages adaptés, de dosimètres à alarme sonore ou de balises « sentinelles » peut concourir à cet objectif. 
Il a été décidé de compléter la base de données RELIR à partir de ces types d'incidents et d'établir de nouvelles fiches.

\section{4. « Produits » de l'atelier}

Trois fiches RELIR ont d'ores et déjà été publiées (et sont disponibles sur le site RELIR). Elles couvrent les incidents suivants (les fiches sont présentées en annexe A) :

- Évacuation incomplète de la zone d'exclusion.

- Incident lors d'une séance de formation.

- Incidents consécutifs à un balisage défaillant.

Deux autres fiches devraient être prochainement proposées, relatives à un franchissement volontaire de balisage et à un blocage du porte-source dans la gaine d'éjection.

En parallèle, une synthèse des leçons issues des incidents recensés a été élaborée. Les participants de l'atelier ont retenu les propositions suivantes :

- Organiser les tirs gammagraphiques en tenant compte des conditions de travail (activités connexes, facteur humain...).

- Effectuer un balisage exhaustif de la zone d'intervention et assurer la maîtrise de la zone balisée au cours des tirs.

- Assurer une utilisation correcte des appareils de gammagraphie.

- Assurer une maintenance régulière des équipements de gammagraphie.

- Contrôler régulièrement le bon fonctionnement des dosimètres et des radiamètres, ainsi que celui des alarmes lumineuses et sonores.

- Vérifier le port (obligatoire) des dosimètres personnels par les opérateurs.

- Disposer d'un endroit sûr et non exposé pour stocker les dosimètres et les radiamètres.

- Réaliser les contrôles radiologiques appropriés pendant et après chaque tir gammagraphique afin d'identifier d'éventuels problèmes et de limiter les expositions incidentelles.

- Assurer la formation des opérateurs et des PCR et instaurer des séances de rappel.

- Rédiger des procédures d'urgence.

Un document complet est disponible en annexe $\mathrm{B}$. Il a vocation à compléter, entre autres, le contenu des séances de formation. Il est à souligner que ces leçons ne concernent pas uniquement les opérateurs de contrôles des entreprises intervenantes, elles s'adressent également à leur hiérarchie ainsi qu'aux donneurs d'ordre (entreprises utilisatrices) et aux personnes compétentes en radioprotection (des entreprises intervenantes et utilisatrices). 


\section{Pérennisation des travaux engagés}

Le domaine des tirs gammagraphiques reste l'un des secteurs où les expositions professionnelles et les risques d'incidents sont les plus élevés : l'analyse et la valorisation du retour d'expérience des événements sont donc primordiales. Suite aux travaux de l'atelier, un membre a accepté de devenir modérateur du réseau RELIR dans le domaine de la gammagraphie industrielle. Sa participation permettra de tenir à jour la base de données (en facilitant la rédaction de nouvelles fiches si un nouveau type d'incident intervient) et de qualifier la pertinence et la représentativité des incidents recensés dans la base de données. Elle sera un moyen de garantir l'intérêt des fiches et leur utilité dans les séances de formation.

\section{Annexe A. Fiches RELIR}

\section{A.1. Évacuation incomplète de la zone d'exclusion lors de contrôles non destructifs}

\section{A.1.1. Circonstances}

L'évacuation incomplète d'une zone balisée avant contrôle radiographique est généralement liée à la concomitance de deux circonstances, il s'agit :

- du volume important de la zone d'exclusion,

- de la présence d'intervenants sur des chantiers parallèles.

Dans ces conditions, le balisage est souvent posé alors que des intervenants sont déjà dans l'espace amené à devenir zone d'exclusion. Ils n'ont alors pas conscience de l'évolution de la situation et n'appréhendent donc pas toujours les risques qu'ils encourrent à rester à leur poste de travail. Dans les faits, le, ou les individus peuvent ainsi être considérés comme « prisonniers ». Par la suite, ils peuvent se trouver à proximité de la source, pendant le contrôle radiographique, sans avoir eu connaissance de la réalisation du tir (en effet, ils n'ont franchi aucun balisage).

Ce type d'événement est caractéristique d'un manque de maîtrise de la zone balisée.

Les deux exemples qui suivent sont significatifs de telles situations où la zone d'exclusion - une salle des machines - est un vaste bâtiment industriel dont les dimensions (longueur $=$ env. 100 mètres, largeur et hauteur $=$ env. 40 mètres) ne facilitent pas l'évacuation avant le début des contrôles radiographiques. 


\section{A.1.1.1. Exemple 1}

En sortant d'un bungalow ${ }^{14}$ installé en salle des machines, deux intervenants constatent qu'ils se trouvent à l'intérieur d'une zone balisée pour la réalisation de contrôles radiographiques, alors que les tirs sont en cours.

\section{A.1.1.2. Exemple 2}

Deux intervenants réalisent un usinage sur un robinet alors qu'ils se trouvent à l'intérieur de la zone d'exclusion pour un tir radiographique. Ils prennent conscience de leur situation lors de la diffusion d'un message sonore préalable au début du tir. Ils évacuent immédiatement leur chantier, ceci quelques minutes seulement avant l'éjection de la source.

Des mesures sur le terrain ont permis de vérifier l'absence de débit de dose sur le chantier et le chemin qu'ils ont emprunté lors de leur évacuation dans le cas où la source aurait été éjectée prématurément.

\section{A.1.2. Conséquences radiologiques}

\section{A.1.2.1. Conséquences réelles}

Dans l'exemple 1, chaque intervenant a reçu une dose de $0,04 \mathrm{mSv}$.

Dans l'exemple 2, aucune dose n'a été intégrée compte tenu de l'évacuation de la zone par les intervenants avant éjection de la source.

\section{A.1.2.2. Conséquences potentielles}

L'exemple 1 aurait pu conduire à l'intégration d'une dose de 0,5 mSv correspondant à toute la durée des tirs.

Pour l'exemple 2, l'absence de débit de dose sur le chantier et le chemin d'évacuation dans la situation « source éjectée » conduit à des doses nulles.

\section{A.1.3. Leçons à tirer}

Comme mis en avant dans le premier paragraphe, plus la zone d'exclusion est importante, plus elle est difficile à maîtriser. En conséquence, lorsque que l'installation le permet, il est nécessaire de définir une zone d'exclusion la plus réduite possible.

Pour favoriser et garantir l'absence d'intervenants dans la zone d'exclusion :

- Il est nécessaire de coordonner et de planifier l'ensemble des activités, y compris les contrôles radiographiques, pour que tous les intervenants aient connaissance

14 Les dimensions de la salle des machines permettent d'héberger ce type de bungalow sans difficulté. 
des activités connexes pouvant les impacter et que des dispositions soient mises en œuvre en fonction des risques identifiés. La réalisation de tirs radiographiques sous-tend en effet une collaboration étroite entre entreprise intervenante et entreprise utilisatrice (articles 237-1 à 237-28 du code du travail).

- Il est important de mener une analyse de risques pour les contrôles radiographiques, puis de définir et de mettre en œuvre les parades correspondantes (programmation des contrôles radiographiques sur le weekend, inspection des locaux isolés ou borgnes pour s'assurer qu'il n'y a personne, mise en place d'un surveillant de tir pour renforcer la maîtrise de la zone...).

- Il est recommandé d'informer préalablement au tir de l'imminence de celui-ci, via les réseaux de sonorisation internes des installations, lorsqu'ils existent.

Par ailleurs, l'entreprise utilisatrice peut contribuer à l'évacuation de la zone en déployant des ressources à cet effet auprès des opérateurs de tir.

\section{A.2. Incident lors d'une séance de formation}

\section{A.2.1. Circonstances}

Lors d'une séance de formation au contrôle gammagraphique, Monsieur A, stagiaire, oublie de mettre en place le raccord à boule permettant de relier le câble de la télécommande au porte-source. Il branche donc directement les deux accessoires. Lorsqu'il veut éjecter la source (Ir-192, 740 GBq), le porte-source se bloque immédiatement après un tour de manivelle, à environ $25 \mathrm{~cm}$ du conteneur du gammagraphe. Monsieur A se retrouve alors dans l'impossibilité de ramener le porte-source en position de stockage.

Monsieur A appelle son responsable, Monsieur B, qui est aussi la personne compétente en radioprotection (PCR). Celui-ci vérifie d'abord qu'il n'est pas nécessaire d'agrandir la zone de balisage puis prend l'initiative de résoudre seul le problème.

Sachant, grâce au nombre de tours de manivelle effectués par le stagiaire, la distance entre le porte-source et le gammagraphe, Monsieur B estime la position de la source et conçoit son intervention de façon à en rester le plus éloigné (par exemple, pour ne pas poser ses mains au niveau de la source). Il décide ainsi d'attraper la gaine d'éjection à environ 1,5 m du gammagraphe puis de lever énergiquement l'ensemble "gaine et gammagraphe » afin de faire redescendre par gravité le porte-source en position de stockage. Monsieur B répète cette manœuvre plusieurs fois grâce à des essais à blanc (sans source) chronométrés. Il intervient ensuite et réussit à récupérer la source. 


\section{A.2.2. Conséquences radiologiques}

Monsieur B portait des dosimètres passif et actif ainsi qu'un dosimètre électronique au poignet. Son temps d'exposition a été très court (quelques secondes) : les doses qu'il a intégrées lors de cette intervention sont donc faibles : $70 \mu \mathrm{Sv}$ corps entier et $110 \mu \mathrm{Sv}$ pour une main. Notons cependant que le débit de dose à $1,5 \mathrm{~m}$ de la source était approximativement de $45 \mathrm{mSv} / \mathrm{h}$.

Le stagiaire était classé catégorie A et portait également ses dosimètres. L'incident s'est déroulé dans un blockhaus : le stagiaire se situait à l'extérieur lors de l'éjection de la source, il n'a donc pas été exposé.

\section{A.2.3. Leçons}

Lors de la manipulation d'un gammagraphe, l'opérateur ne doit pas forcer une connexion entre deux éléments, ce qui a certainement été fait dans cet exemple pour relier directement le câble de la télécommande au porte-source.

Quels que soient le type et le lieu des incidents, les consignes de sécurité précisent qu'un opérateur ne doit pas tenter de les résoudre seuls. En cas d'incident complexe, il est recommandé de faire directement appel au constructeur des gammagraphes ou à l'IRSN (Institut de radioprotection et de sûreté nucléaire) qui disposent des ressources et des compétences nécessaires pour intervenir en situation incidentelle.

Si un tel incident a lieu en dehors d'un blockhaus (sur la voie publique, dans un lieu public ou dans une entreprise non spécialisée), il est recommandé de recourir, en plus de l'IRSN et du fabricant, à d'autres services. Dans certains cas, la situation est en effet susceptible d'évoluer en situation d'urgence radiologique.

Ces services sont alors généralement représentés par les services d'incendie et de secours et les forces de police ou de gendarmerie. Ils assurent, au départ, les premières mesures conservatoires et se chargent d'aviser les autorités de police administratives générales que sont le Maire et le Préfet, et l'ASN. Par la suite, ils concourent à la solution de l'incident.

En cas d'incident, il est essentiel de localiser la source. La réalisation de contrôles radiologiques (mesure des débits de dose) reste le moyen le plus précis et le plus sûr. De plus, lorsque l'intervention a été définie et si cela est possible, une bonne pratique consiste à réaliser plusieurs essais à blanc (sans source) afin d'optimiser les gestes et les temps d'exposition.

Cet incident souligne le besoin de contrôler les connaissances des opérateurs et d'accompagner les jeunes intervenants lors de leurs premières manipulations. Des 
procédures internes au sein des entreprises de contrôle pourraient être définies en ce sens.

Lors des formations, il peut aussi être utile de former les stagiaires aux comportements à adopter face aux incidents. Ici, Monsieur A a bien réagi puisqu'il a directement contacté la PCR et n'a pas cherché à récupérer la source.

\section{A.3. Incidents consécutifs a un balisage défaillant}

\section{A.3.1. Circonstances}

Ces incidents peuvent être définis de la façon suivante : le balisage est défaillant, un accès n'est pas balisé et permet de pénétrer à l'intérieur de la zone d'exclusion. Le, ou les, opérateurs peuvent alors se rapprocher de la source pendant le contrôle radiographique et ainsi être exposés aux rayonnements.

Ce type d'événement est caractéristique d'un manque de maîtrise du balisage. Plusieurs causes peuvent être à l'origine de ce type de situation :

- des plans de locaux incomplets ou imprécis,

- une pose incomplète du balisage,

- un non-respect du plan de balisage d'origine.

Les exemples ci-dessous illustrent chacune de ces trois familles.

\section{A.3.1.1. Exemple 1 dans une installation industrielle}

Dans le cadre d'un contrôle radiographique sur un circuit d'incendie dans une station de pompage d'eau en bord de rivière, une absence de balisage est constatée en bas d'une échelle permettant d'accéder du rez-de-chaussée à un niveau supérieur situé dans la zone d'exclusion.

Les plans de la station de pompage n'étaient pas suffisamment précis, ils n'identifiaient pas tous les cheminements possibles, dont les accès potentiels par les échelles. C'est une vérification indépendante du balisage, par le service compétent en radioprotection de l'installation industrielle, qui a permis de détecter cet écart avant le début du tir gammagraphique. L'autocontrôle réalisé par le chargé de travaux de l'entreprise de contrôle radiographique n'avait pas permis de détecter l'erreur de balisage.

\section{A.3.1.2. Exemple 2 dans une salle des machines}

Pour réaliser une campagne de tirs gammagraphiques dans une salle des machines, celle-ci a été totalement isolée (interdite d'accès). 
Pourtant, le non-respect du plan de balisage permettait potentiellement d'accéder à la zone d'exclusion par un escalier situé à l'extérieur de la salle des machines mais desservant chaque étage de celle-ci. L'accès à cet escalier aurait donc dû être interdit et signalé par une balise et un gyrophare. Dans les faits, seule la porte d'accès à la salle des machines située au rez-de-chaussée dans la cage d'escalier était balisée : on pouvait donc accéder à la zone d'exclusion par les étages sans franchir ni voir de balisage.

C'est l'attitude interrogative d'une personne ayant emprunté l'escalier qui a permis de détecter l'absence du balisage. Cette personne a trouvé incohérent de rencontrer un balisage au rez-de-chaussée et de ne pas en voir aux étages supérieurs.

\section{A.3.1.3. Exemple 3 dans un bâtiment industriel}

Lors d'une activité de contrôle de soudures, le plan de balisage n'a pas été respecté par l'opérateur de tir : ni banderole, ni balise lumineuse n'a été posée sur deux échelles qui permettaient d'accéder à une pièce côtoyant le local où des tirs gammagraphiques étaient en cours.

Cet écart a été constaté lors d'une activité de contrôle sur des chantiers à proximité de ces deux accès potentiels.

\section{A.3.2. Conséquences radiologiques}

\section{A.3.2.1. Conséquences réelles}

Aucune : personne, à aucun moment, ne s'est trouvé dans la zone d'exclusion. Aucune dosimétrie n'a été intégrée.

\section{A.3.2.2. Conséquences potentielles}

Dans les deux premiers cas présentés ci-dessus, une intrusion involontaire aurait pu conduire au dépassement d'une limite de dose réglementaire $(1 \mathrm{mSv})$ pour la ou les personnes qui auraient emprunté les accès non balisés.

Dans le troisième exemple, une personne présente dans le local accessible par les échelles n'aurait pas pu s'approcher de la source, la configuration du local l'interdisant. Dans l'hypothèse où elle aurait séjourné dans ce local pendant toute la durée du contrôle radiographique (3 heures), elle aurait intégré une dose maximale de 1,7 mSv.

\section{A.3.3. Leçons à tirer}

Dans la mesure du possible, la mise en place du balisage doit être vérifiée par le chargé de travaux de l'entreprise de contrôle radiographique (autocontrôle) ainsi que par une entité indépendante des opérateurs de tirs. 
L'attitude interrogative de toute personne amenée à côtoyer les tirs radiographiques peut aussi aider à la vérification de la mise en place des balisages, et dans certains cas, éviter des incidents ou accidents d'exposition. Une formation ou, à défaut, une information minimale sur les risques potentiels inhérents à une intrusion en zone de tir, doit donc être assurée pour tous les intervenants non directement concernés par ce type d'opération.

Par ailleurs, il est essentiel de disposer de plans de balisage de qualité, élaborés à partir de plans de l'installation identifiant les conditions réelles de l'environnement de travail. Cela sous-tend la mise à disposition de plans lisibles et de dimension adaptée pour garantir cette lisibilité ainsi que l'adoption de représentations standardisées (pour les portes, trappes, échelles, ascenseurs...).

Rappelons enfin que la réalisation de tirs radiographiques sous-tend une collaboration étroite entre entreprise intervenante et entreprise utilisatrice (articles 237-1 à 237-28 du code du travail).

\section{Annexe B. Leçons issues du REX des incidents}

Ce document propose une synthèse des leçons issues des incidents recensés au cours des deux réunions de l'atelier : il vise à être le plus exhaustif possible quant aux types de leçons. Il a notamment vocation à être utilisé lors de séances de formation des opérateurs et des personnes compétentes en radioprotection des entreprises intervenantes et utilisatrices.

\section{B.1. Organiser les tirs gammagraphiques en tenant compte des conditions de travail (activités connexes, facteur humain...)}

Les tirs gammagraphiques doivent être organisés en tenant compte des autres activités industrielles et dans le respect des plannings.

Il est important de prendre en compte les conditions de travail difficiles, voire hostiles des radiologues dans l'organisation des tirs gammagraphiques. La charge de travail doit être compatible avec l'attention nécessaire à la réalisation du tir. 


\section{B.2. Effectuer un balisage exhaustif de la zone d'intervention et assurer la maîtrise de la zone balisée au cours des tirs}

Les plans fournis par les donneurs d'ordre doivent être suffisamment détaillés afin de permettre aux radiologues de baliser de manière exhaustive la zone de tirs.

La zone de tirs doit être correctement balisée afin d'éviter tout accès involontaire dans le périmètre.

\section{B.3. Assurer une utilisation correcte des appareils de gammagraphie}

Lors de chaque nouveau tir gammagraphique, il est nécessaire de respecter les phases suivantes :

- Rentrer la source dans le gammagraphe par la télécommande.

- Vérifier la présence de la source dans le gammagraphe.

- Mesurer le débit de dose avec un radiamètre en rentrant dans le local de tir afin de confirmer la présence de la source dans le gammagraphe.

- Retirer la cassette exposée.

- Repositionner l'embout d'irradiation pour la nouvelle exposition.

- Mettre en place une cassette non exposée.

- Réarmer le gammagraphe.

- Ressortir du local de tir.

- Commander l'éjection de la source du gammagraphe pour le tir suivant.

- Chronométrer le temps d'exposition dont la durée doit être prévue avant chaque série de contrôles.

Dans la mesure du possible, il faut éviter les géométries sinueuses pour le flexible d'éjection car ceci entraîne le plus souvent un blocage du porte-source.

Il est important de joindre une notice d'utilisation à tout matériel.

Pour des opérations particulières comme le remplacement d'une source usagée par une source neuve, il est conseillé que l'opérateur ait à sa disposition des procédures écrites.

Les sources doivent toujours être remises en position de sûreté quand elles ne sont pas utilisées.

Il est important que le radiologue contrôle l'intégrité et la propreté des accessoires avant de réaliser les branchements. Des corps étrangers (sable, rouille) peuvent en effet pénétrer dans les flexibles quand les appareils sont utilisés à l'extérieur ou dans des environnements industriels hostiles. 


\section{B.4. Assurer une maintenance régulière des équipements de gammagraphie}

Les équipements de gammagraphie doivent être inspectés visuellement lors de chaque essai, faire l'objet d'une maintenance régulière et détaillée et être soumis à des contrôles réguliers par des spécialistes.

Les contrôles de maintenance doivent comporter un examen de l'intégrité du conteneur et une inspection des flexibles.

Une maintenance régulière doit être réalisée sur toutes les parties mécaniques de l'appareil.

Il est important de vérifier régulièrement que la source ne fuit pas.

Il est important que les équipements de gammagraphie fassent l'objet d'une maintenance régulière et d'un nettoyage a minima annuel.

Les fournisseurs et les constructeurs d'équipements doivent spécifier les procédures et les produits chimiques à utiliser pour nettoyer leurs appareils (certains peuvent nuire à l'intégrité des conteneurs).

Lorsqu'un appareil est défectueux, les opérateurs doivent le signaler à leur employeur (qui doit alors le renvoyer à son fournisseur pour une révision) et non pas essayer de le réparer sommairement.

\section{B.5. Contrôler régulièrement le bon fonctionnement des dosimètres et des radiamètres, ainsi que celui des alarmes lumineuses et sonores}

Les dosimètres personnels et les radiamètres permettant de contrôler les débits de dose doivent faire l'objet d'une maintenance régulière.

Des contrôles réguliers des systèmes d'alarme et des appareils de mesure sont nécessaires afin de s'assurer que ceux-ci restent opérationnels.

Les dosimètres utilisés doivent être équipés d'alarmes sonore et lumineuse, sur des critères de dépassement d'une contrainte opérationnelle de dose et de débit de dose. Leur bon fonctionnement doit être vérifié périodiquement.

Les compagnies qui utilisent des alarmes automatiques doivent régulièrement vérifier leur fonctionnement et leur position. Par exemple, les alarmes lumineuses 
doivent toujours être placées dans des lieux visibles. De même, les alarmes sonores doivent être suffisamment fortes pour être entendues dans divers endroits (particulièrement, si des protections auditives doivent êtres portées pour d'autres raisons).

\section{B.6. Vérifier le port (obligatoire) de dosimètres personnels par les opérateurs}

Pendant les séances de gammagraphie, les opérateurs doivent impérativement porter leurs dosimètres et laisser brancher les détecteurs sonores.

Le port du dosimétrique électronique est obligatoire et permet d'alerter les opérateurs en cas d'exposition incidentelle.

Des dosimètres nominatifs doivent être portés.

Les opérateurs doivent allumer leur dosimètre dès qu'ils travaillent à proximité d'une zone de tirs.

Lorsqu'il y a un risque de contamination interne et qu'il est nécessaire de porter des équipements particuliers de protection, les opérateurs doivent avoir à leur disposition des surtenues équipées de fenêtres translucides permettant de laisser visible leur dosimètre.

\section{B.7. Disposer d'un endroit sûr et non exposé pour stocker les dosimètres et les radiamètres}

Les employeurs doivent fournir des lieux appropriés pour le stockage des dosimètres.

\section{B.8. Réaliser les contrôles radiologiques appropriés pendant et après chaque tir gammagraphique afin d'identifier d'éventuels problèmes et de limiter les expositions incidentelles}

À la fin d'une séance de gammagraphie, les opérateurs sont tenus de contrôler la bonne réintroduction de la source radioactive dans son conteneur à l'aide d'un radiamètre par exemple.

Il est important de vérifier la présence de la source dans son conteneur en fin de manipulation et de suivre « son parcours » en continu lors des manipulations. 


\section{B.9. Assurer la formation des opérateurs et des PCR et instaurer des séances de rappel}

Il est nécessaire de remettre à jour la formation des PCR (personnes compétentes en radioprotection) régulièrement.

Il est nécessaire de vérifier périodiquement les capacités des opérateurs et de mettre en place des séances de rappel d'utilisation des matériels.

Les employés doivent être formés à l'utilisation des dosimètres et des appareils de mesures.

Les opérateurs doivent être sensibilisés à la dosimétrie « extrémités ».

Les opérateurs doivent connaître les systèmes d'alarme et de sécurité. Des notices expliquant la signification des alarmes doivent être placées dans des lieux stratégiques.

Les formations doivent insister sur les forts débits de dose enregistrés à de courtes distances des sources et sur la nécessité d'éviter les contacts proches.

Les formations doivent former les opérateurs et les PCR à «bien réagir » en situation incidentelle.

\section{B.10. Rédiger des procédures d'urgence}

Les procédures d'urgence sont une part importante des programmes de radioprotection des entreprises.

Pour des sources de haute activité, l'attention doit être portée à l'élaboration de procédures qui permettent de récupérer les sources bloquées sans conduire à des niveaux d'exposition trop importants.

Les procédures d'urgence doivent indiquer que certaines situations peuvent demander l'intervention de personnel plus qualifié que le personnel interne. Elles doivent identifier des critères à partir desquels une intervention externe doit être demandée.

Les kits d'urgence doivent être disponibles sur chaque zone de tir. Les opérateurs ne devraient pas avoir à se rendre dans d'autres locaux pour les récupérer.

La compagnie doit prévoir de mettre en place des exercices afin de former ses employés aux procédures d'urgence. Des équipements supplémentaires peuvent être achetés afin de permettre la mise en place de ces plans. 


\section{B.11. Autres : Transport}

Les conteneurs de source doivent toujours être transportés avec l'obturateur fermé et sans la clé.

Il est déconseillé de transporter des conteneurs de source en même temps que d'autres objets encombrants.

\section{RÉFÉRENCES}

Croft J.R., Thomas G.O., Walker S., Williams C.R. (1999) IRID: Ionising Radiations Incident Database - First review of cases reported and operation of the database.

Vaillant L., Bataille C., Croüail P., Lefaure C. (2006) Retour d'Expérience sur Les Incidents Radiologiques : le système RELIR, Rapport CEPN n²97.

European ALARA Network, Summary and recommendations from the 5th Workshop on "Industrial Radiography: Improvements in Radiation Protection”, Rome, 2001.

AIEA (1998) Leçons issues des accidents en gammagraphie industrielle, Safety Report Series No. 7. AIEA (1999) Radioprotection et sûreté en gammagraphie industrielle, Safety Reports Series No. 13. 